Journal of Clinical and Nursing Research

Research Article

\title{
Correlation Analysis of Intervertebral Disc Degeneration with HTRA1 and HAPLN1 Gene Polymorphisms
}

\author{
Tuanmao Guo ${ }^{1}$, Yanli Xing ${ }^{2 *}$, Zhongning Chen ${ }^{1}$, Haiyun Zhu ${ }^{1}$ \\ ${ }^{1}$ Department of Orthopedics, Xianyang Central Hospital, Xianyang 712000, China \\ ${ }^{2}$ Department of Pharmacy, Xianyang Central Hospital, Xianyang 712000, China
}

Funding: This work was supported by the Project of the Department of Science and Technology in Shaanxi Province (2020JM-702).

\begin{abstract}
The lumbar spine, an important part of the body's motor mechanism, is more susceptible to damage as it bears most of the body's load. Age can cause clinical manifestations such as neurological impairment, back and leg pain in the lumbar spine. External forces result in nucleus pulposus out, destruction of the intervertebral disc fibrous ring, and gradual aging and damage. Lumbar degenerative change is a common middle-aged and old-aged disease, and its clinical symptoms on the initial stage are not obvious, but it becomes more and more serious as they get older. Patients with severe lumbar degenerative changes will appear symptoms such as urinary and fecal incontinence, lower extremity numbness, back pain, and sexual dysfunction. The main reason for back pain and leg pain is the degenerative changes in the lumbar intervertebral discs, at the same time which also leads to patients' lumbar instability. This study focuses on the correlation analysis of intervertebral disc degeneration with HTRA1 and HAPLN1 gene polymorphisms.
\end{abstract}

Keywords: HTRA1; HAPLN1; Intervertebral disc degeneration

Publication date: September, 2020

Publication online: 30 September, 2020

* Corresponding author: Yanli Xing, 18292936356@163.com

\section{Introduction}

Intervertebral disc degeneration (IDD) is the pathological basis for herniated discs, spinal stenosis, and other related conditions, and it is one of the leading causes of back and leg pain. The reduction of proteoglycans in the nucleus pulposus tissue of the intervertebral disc and the appearance of fibrous ring fissures lead to the protrusion of the nucleus pulposus tissue through the fissures of the fibrous ring, causing a corresponding immune-inflammatory response and simultaneous compression of the spinal cord and nerve roots by the protruding nucleus pulposus, leading to symptoms of low back and leg pain.

The intervertebral disc is composed of the upper and lower cartilaginous endplates, the fibrous annulus, and the central nucleus pulposus, which play a vital role in weight-bearing and movement of the spine. IDD is associated with many factors, mainly including inflammatory causes, biomechanics, oxidative stress, apoptosis, dystrophic pathways, matrix metalloproteinases (MMPs), and tissue inhibitors of metalloproteinases. The inflammatory response is one of the important factors in IDD, in which interleukin (IL), tumor necrosis factor-alpha (TNF- $\alpha$ ), prostaglandin E2 (PGE2), nitric oxide (NO) and nitric oxide synthase (NOS) all play a very important role in the overall development and progression of IDD.

\section{Materials and Methods}

\subsection{Materials}

498 postmenopausal Chinese women, aged (67.4 \pm 7.5$)$ years, who underwent physical examinations at Dingzhou People's Hospital from April 2015 to December 
2018, were selected as the subjects. Exclusion of subjects with endocrine disorders such as hyperthyroidism, hyperparathyroidism or diabetes mellitus, liver disease, kidney disease, use of drugs known to affect bone metabolism (e.g. corticosteroids, anticonvulsants, heparin sodium), or an uncommon history of gynecological problems. Patients with severe hip and knee arthritis. The eligibility of the subjects was determined by questionnaire and physical examination. A trained nurse collected $5 \mathrm{Ml}$ of a venous blood sample from the subject using a vacuum cannula containing EDTA anticoagulant, mixed it up, and placed it at $-80^{\circ} \mathrm{C}$ for freezing, and extracted genomic DNA. Informed consent was obtained and signed by all subjects before the commencement of the study. The study was approved by the ethics committee of Dingzhou People's Hospital.

\subsection{Methods}

\subsubsection{Radiographic grading of intervertebral disc degeneration}

Routine planar x-rays of the thoracic and lumbar spine were obtained for all subjects in transverse, anterior, and posterior projections. Both lateral protrusion and supine (A-P) projections were taken at a constant focus film distance of $1.5 \mathrm{~m}$. The severity of intervertebral disc degeneration was assessed by the grading scale of intervertebral disk level(T4-T5 to L4-L5) or by the semi-quantification of vertebrae (T4-L4), including osteophyte formation, endplate sclerosis, and spinal stenosis: osteophyte score: grade 0 , no ectopic bone formation; grade 1, osteonecrosis $<2 \mathrm{~cm}$; grade 2 , osteonecrosis $>2 \mathrm{~cm}$; grade 3 , osteonecrosis $>5 \mathrm{~cm}$; endplate sclerosis score: grade 0 , normal, no degeneration; grade 1, edema; grade 2, fatty degeneration or ischemic necrosis of bone marrow; grade 3 , osteosclerosis; spinal stenosis score: grade 0 , no spinal stenosis; grade 1 , mild stenosis; grade 2, severe stenosis. Intervertebral disc degeneration was assessed according to the reported scoring system. The sum of each degree of T4-L4 vertebral endplate sclerosis and the sum of the T4-T5 to L4L5 intervertebral disc levels were used as the endplate sclerosis and intervertebral space narrowing scores.

\subsubsection{HTRA1 gene single nucleotide polymorphism}

Samples were genotyped by TaqMan genotyping assay using ABIPrism7500 Sequence Detection System (AppliedBiosystems), including forward and reverse sequence-specific primers and two TaqManMGB probes for allele detection. The TaqMan probe contains a flu- orescent dye at the 5' end and a quencher at the 3' end. During the PCR cycle, the two TaqMan probes competitively hybridized to specific sequences of the target DNA, and the reporter group was separated from the quenching group, resulting in increased fluorescence of the reporter group. Three genotypes of single nucleotide polymorphisms were identified by monitoring the fluorescence levels of the PCR products.

\subsection{Main observation indexes}

Osteophyte score, final plate sclerosis score, and intervertebral disc stenosis score in all subjects.

\subsection{Statistical analysis}

The data of this study were analyzed by SPSS20.0 software. $p<0.05$ was considered statistically significant.

\section{Results}

\subsection{The number of participants}

All 498 subjects were enrolled in the analysis of the results.

\subsection{Relationship between HTRA1 gene promoter polymorphism and spinal stenosis}

The genotype of the 1rs11200638 single nucleotide polymorphism in the promoter region of the HTRA1 gene from 498 subjects was analyzed using TaqMan methods. In these postmenopausal women, 178 were GG homozygotes, 222 were GA heterozygotes, and 98 were AA homozygotes. The allele frequencies of this single nucleotide polymorphism were in HardyWeinberg equilibrium.

Intervertebral disc degeneration parameters including osteophyte formation, endplate sclerosis, and intervertebral stenosis were compared between subjects with at least one $\mathrm{G}$ allele $(\mathrm{GG}+\mathrm{GA})$ and those without the $G$ allele (AA), as shown in Table 1.

Independent-sample t-test analysis showed a significant difference between HTRA1 genotypes and intervertebral stenosis score $(\mathrm{P}<0.001)$, whereas there was no significant difference between endplate sclerosis or osteophyte formation and single nucleotide polymorphism genotypes. By comparing the background data (age, height, and weight) for GG+GA and AA genotypes, a significant difference was observed between HTRA1 genotype and height $(\mathrm{P}=0.001)$, but age or weight was not statistically different between groups.

The correlation between allele frequencies of HTRA1 
Table 1. Comparison of the background and clinical characteristics of subjects with different HTRA1 single nucleotide polymorphism genotypes $(\mathrm{X} \pm \mathrm{S})$

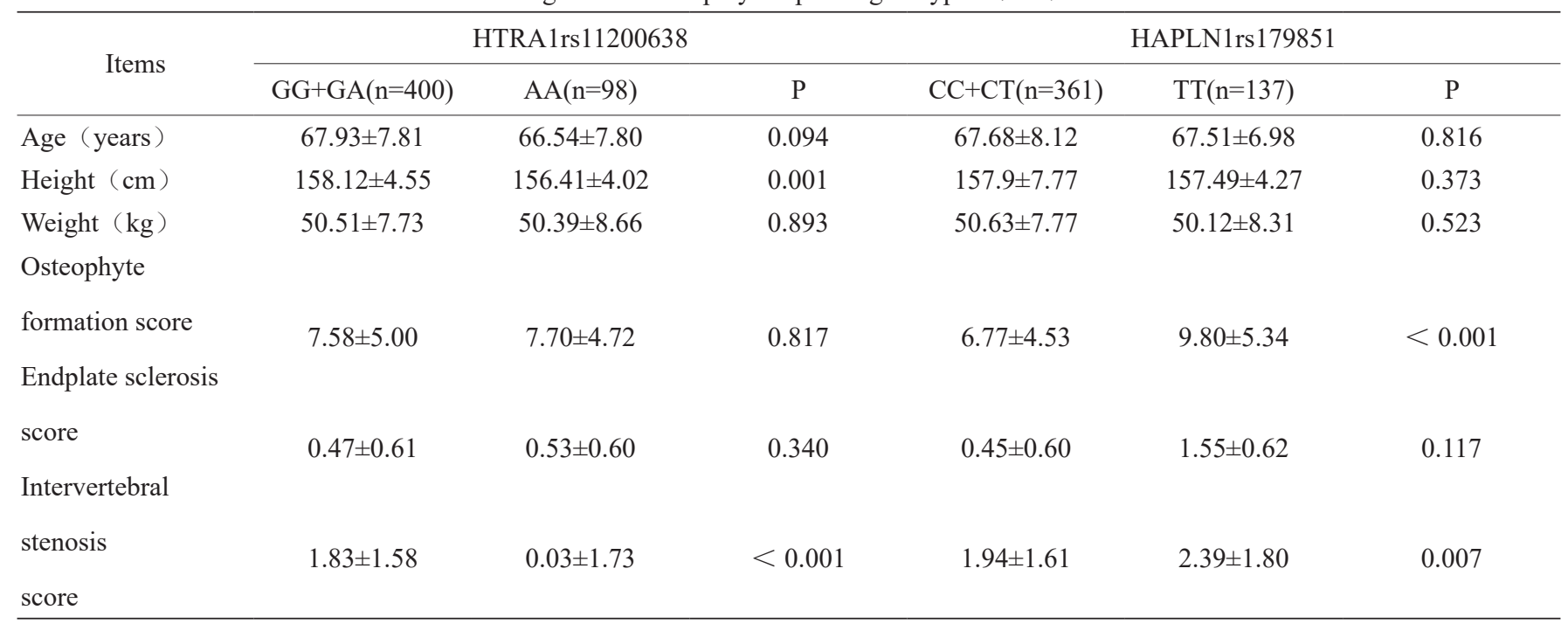

Table 2. Logistic regression analysis of single nucleotide polymorphism genotypes and intervertebral disc degeneration parameters

\begin{tabular}{|c|c|c|c|c|}
\hline \multirow[b]{2}{*}{ Intervertebral disc degeneration parameters } & \multirow[b]{2}{*}{ OR value } & \multicolumn{2}{|c|}{$95 \% \mathrm{Ct}$ value } & \multirow[b]{2}{*}{$\mathrm{P}$} \\
\hline & & Lower limits & $\begin{array}{l}\text { Upper } \\
\text { limits }\end{array}$ & \\
\hline$\geq 4 \quad(\mathrm{n}=96)$ vs $<4(\mathrm{n}=402)$ & 2.347 & 1.419 & 3.883 & 0.001 \\
\hline$\geq 5(\mathrm{n}=49)$ vs $<5(\mathrm{n}=449)$ & 4.874 & 2.633 & 9.023 & $<0.001$ \\
\hline$\geq 6(\mathrm{n}=22) v s<6(\mathrm{n}=476)$ & 4.492 & 1.879 & 10.739 & 0.001 \\
\hline \multicolumn{5}{|l|}{$\begin{array}{l}\text { HAPLN1rs179851 and intervertebral } \\
\text { stenosis }\end{array}$} \\
\hline$\geq 4 （ \mathrm{n}=96)$ vs $<4(\mathrm{n}=402)$ & 0.851 & 0.511 & 1.419 & 0.536 \\
\hline$\geq 5(\mathrm{n}=49)$ vs $<5(\mathrm{n}=449)$ & 1.455 & 0.780 & 2.717 & 0.239 \\
\hline \multicolumn{5}{|l|}{$\begin{array}{l}\text { HAPLN1rs179851 and intervertebral } \\
\text { stenosis }\end{array}$} \\
\hline$\geq 7 （ \mathrm{n}=286) \quad v s<7(\mathrm{n}=212)$ & 2.676 & 1.735 & 4.126 & $<0.001$ \\
\hline$\geq 8(\mathrm{n}=243)$ vs $<8(\mathrm{n}=255)$ & 2.867 & 1.894 & 4.339 & $<0.001$ \\
\hline$\geq 9(\mathrm{n}=202) v s<9(\mathrm{n}=298)$ & 2.885 & 1.923 & 4.328 & $<0.001$ \\
\hline$\geq 10(\mathrm{n}=168) v s<0(\mathrm{n}=330)$ & 3.065 & 2.037 & 4.614 & $<0.001$ \\
\hline
\end{tabular}

single nucleotide polymorphism genotypes and high intervertebral stenosis scores was analyzed by dividing subjects into $\mathrm{G}$ allele-carrying group $(\mathrm{GG}+\mathrm{GA}, \mathrm{n}=400)$ and uncarrying group (AA, $n=98$ ). The age-adjusted results showed that the risk of AA genotype was significantly increased in subjects with an intervertebral stenosis score of $\geq 4$ compared to subjects with an intervertebral stenosis score of $<4$; the risk of AA genotype was also significantly increased in subjects with a score of $\geq 5$ compared to subjects with a score of $<4$; and the risk of AA genotype was also significantly increased in subjects with a score of $\geq 6$ compared to subjects with a score of $<6$, as shown in Table 2 . Thus, the research suggests that genetic variation in the promoter locus of the HTRA1 gene is associated with intervertebral disc degeneration, particularly intervertebral stenosis.

Only the intervertebral disc degeneration parameters that differ significantly from the two single nucleotide polymorphism genotypes(HTRA1rs 11200638 and HAPLN1rs179851) are listed. 


\subsection{The relationship between HAPLN1 gene polymorphism and intervertebral disc degeneration}

Four single nucleotide polymorphisms (5' flank rs975563, intron 1 rs10942332, intron 2 rs179851, and intron 4 rs4703570) of the HAPLN1 gene were genotyped in 498 volunteers. The results showed that the allele frequencies of the four single nucleotide polymorphisms were in Hardy-Weinberg equilibrium.

\section{Discussion}

Lumbar vertebrae degeneration is a common disease of the elderly and its initial clinical symptoms are not obvious, but it will become more and more serious as they get older. Patients with severe lumbar vertebrae degeneration will appear symptoms such as urinary and fecal incontinence, lower limb numbness, back pain, and sexual dysfunction. Degenerative changes in the lumbar intervertebral discs are the main reason for low back or leg pain and lumbar vertebra instability. Imaging examinations in the hospital is the main way to detect the degree of lumbar intervertebral disc degeneration in patients, such as CT and MRI.

This study demonstrates the correlation of functional single nucleotide polymorphisms in the HTRA1 and HAPLN1 genes with the radiological characteristics of intervertebral disc degeneration in the postmenopausal women in China. Women with the AA genotype in the HTRA1 gene single nucleotide polymorphism rs 11200638 and the TT genotype in the HTRA1 gene single nucleotide polymorphism rs179851 have significantly higher intervertebral disc degeneration scores. However, the correlation between these functional single nucleotide polymorphisms and intervertebral disc degeneration in other populations (including males) needs to be demonstrated in further studies. In conclusion, HTRA1 and HAPLN1 genotyping may be beneficial in the prevention and control of intervertebral disc degeneration. HTRA1 and HAPLN1 will be useful molecular targets for the development of new diagnostic markers and therapeutic strategies.

\section{References}

[1] Taotao X, Fei L, Hongting J, et al. Research progress of intervertebral disc degeneration and cell death $[\mathrm{J}]$. China Bone Injury, 2015, 28(7): 673-678.

[2] Meng XY, Xia JL, Ting Y, et al. Mechanism and repair of intervertebral disc degeneration $[\mathrm{J}]$. Chinese Tissue Engineering Research, 2015, 19(11): 1768-1773.

[3] Ge YQ, Zhou XJ, Bao XP, et al. Application of 3.0T MR IDE$\mathrm{AL}$ and T2 mapping imaging in lumbar intervertebral disc degeneration[J]. Chinese Journal of CT and MRI, 2015, 13(2): $81-83+87$. 